\title{
Exploring the Applicability of Elaborateness and Indirectness in Dialogue Management
}

\author{
Louisa Pragst, Wolfgang Minker and Stefan Ultes
}

\begin{abstract}
In this paper, we investigate the applicability of soft changes to the system behaviour, namely changing the amount of elaborateness and indirectness displayed. To this end, we examine the impact of elaborateness and indirectness on the perception of human-computer communication in a user study. Here, we show that elaborateness and indirectness influence the user's impression of a dialogue and discuss the implications of our results for adaptive dialogue management. We conclude that elaborateness and indirectness offer valuable possibilities for adaptation and should be incorporated in adaptive dialogue management.
\end{abstract}

\section{Introduction}

Spoken dialogue systems are employed under a number of varying conditions (e.g. the amount of ambient noise or different user states). The user experience when interacting with such a system may be improved if those conditions are taken into account, e.g. by the Dialogue Manager (DM).

In a dialogue system, the DM is the component responsible for keeping the dialogue state updated and selecting the system's next action. There are several DM architectures that enable adaptivity (e.g. [3, 5, 9, 10]), often employing hard adaptation approaches. Such approaches are characterised by utilising dedicated system

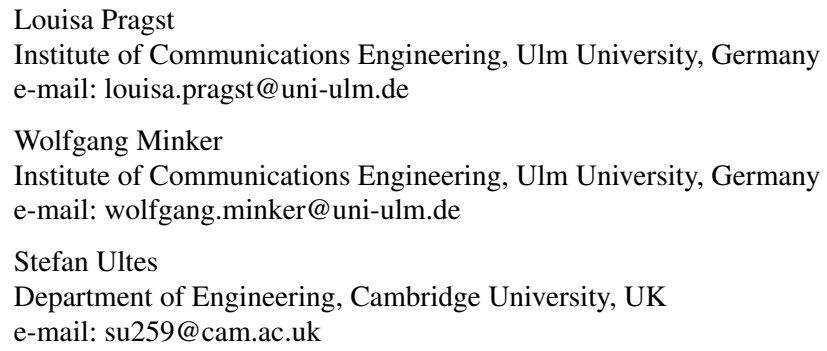


actions to deal with certain conditions, such as reacting to a angry user by asking what is wrong. Gnjatović et al. [5], for example, present a DM that adjusts the kind of support given to the user to their emotional state, and an in-car dialogue system implemented by Kousidis et al. [7] adapts to situations that require the full attention of the driver by pausing the conversation entirely. Saerbeck et al. [8] vary the social supportiveness of a robotic tutor by using motivational sentences such as 'It was not easy for me either'. Similarly, user studies on adaptive dialogue management often focus on hard adaptations: Jaksic et al. [6] employ fixed phrases as response to the user's emotion, effectively leaving two ways to react: apologising and acknowledging positive emotion. Bertrand et al. [2] offer four kinds of emotional feedback: thankfulness, praise, calm and motivate.

In contrast, soft adaptation is only rarely considered. It is characterised by keeping the propositional content of a system action and changing only the way it is presented, e.g. by phrasing statements more politely if the user is angry. Employing soft changes can offer benefits: it is less obvious to the user than a hard adaptation as it is not directly addressing the cause of the adaptation. Asking the user what is wrong will notify the user of the fact that the dialogue system is reacting to their mood. Continuing with the intended statement, just more politely, can appear a seamless continuation of the dialogue. Additionally, hard adaptations are more likely to disrupt the conversation flow by bringing up a new topic (in this case the emotional state of the user). Finally, hard adaptations can become repetitive if employed too often. Asking the user what is wrong is a viable behaviour once anger is detected, but if the user stays angry it is not advisable to repeat this action within the next few exchanges. In contrast, a soft adaptation may be employed over the course of several exchanges as it can be applied to different statements. Being more polite does not become repetitive if the propositional content of the system actions changes. André et al. [1] have proposed the adjustment of the politeness level for adaptation. While the robotic tutor of Saerbeck et al. [8] uses hard adaptation in the form of fixed motivational sentences, it also employs soft adaptation, e.g. on the wording level by using either 'you' or 'we' to reflect the level of closeness between tutor and student. In this paper, we investigate the applicability of two further means of soft adaptation: the level of elaborateness and indirectness (EI).

Elaborateness refers to the amount of additional information provided to the user. A high level of elaborateness could for example result in giving a weather forecast for the next few days when the user asks for the current weather, while with a low elaborateness only the requested information would be given.

The level of indirectness describes how concretely information is addressed by a speaker. For instance, a direct response to a user request about the current weather would be an accurate description of the weather, e.g., 'It is raining'. An indirect response would be the advice to take an umbrella. In the latter case, the weather is not mentioned directly but can be inferred from the given information.

In the following, we show that EI should be incorporated in adaptive dialogue management as they offer valuable possibilities for adaptation. To this end, we present a user study that answers the following questions: does the perception of 


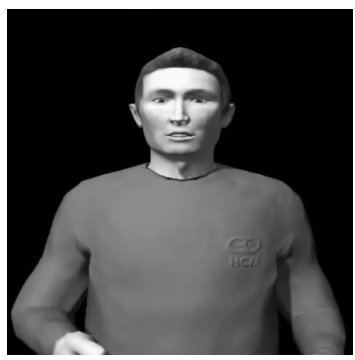

(a) The virtual avatar [4] that was used in our user study.

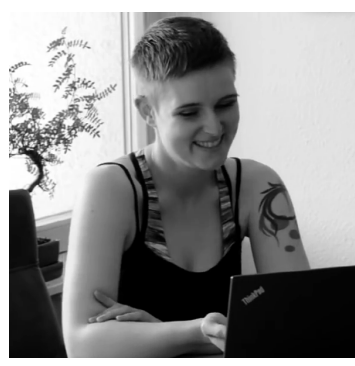

(b) Louisa being involved in the conversation.

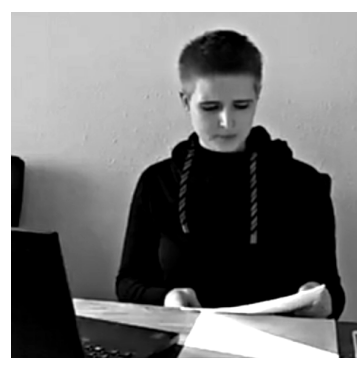

(c) Louisa being distracted from the conversation.

Fig. 1 Screenshots of the videos used in the user studies.

a dialogue change with different EI-levels and is the effect of the EI-level dependant on the current situation of the user.

The remainder of the paper is structured as follows: Section 2 describes the setup and results of our user study. The implications for adaptive dialogue management are discussed in Section 3. Finally, we draw our conclusion in Section 4.

\section{User Study}

The goal of our study is to assess the effect of EI on the perception of a dialogue under different conditions. To this end, we utilise two semantically similar dialogues that differ with regard to the level of EI used by the dialogue system. If the assessment of the two dialogues differs, this supports the assumption that EI can be used in adaptive dialogue management.

\subsection{Study Design}

Our study aims to answer two research questions: does the assessment of a dialogue change with different EI-levels and is the effect of the EI-level dependant on the current situation of the user. This results in two independent variables: the EI-level of the dialogue, instantiated with the two levels low and high, and the situation of the user, with the levels involved and distracted. Differences between the EI-levels signify that participants are receptive to the changes resulting from changing the level of EI and have a preference. If that is the case, EI should be considered when designing the system behaviour with this preference in mind. Furthermore, if the assessment of EI-levels changes in different situations, there is no fixed preference for one specific EI-level and, therefore, EI can be used for adaptation. 
Four conditions result from the independent variables: high EI/involved user, low EI/involved user, high EI/distracted user and low EI/distracted user. For each condition, a video showing a dialogue between a human and a virtual agent was recorded ${ }^{1}$, $2,3,4$. In the videos, a caregiver, called Louisa, interacts with the dialogue system by a virtual avatar called Christian. She suspects that her patient Mr. Smith does not drink enough and expects help from the dialogue system. Screenshots of Christian and Louisa as depicted in the videos can be found in Figure 1. While our videos presented Christian as a real dialogue system, his behaviour followed scripts that were handcrafted for this study. No actual dialogue system was used in the recordings of the videos. The dialogues in the videos differ slightly between the two EI-levels: Christian uses either a high or a low level of EI. A transcription of the dialogues is provided in the Appendix. Additionally, Louisa is depicted as either involved or distracted in the videos, depending on the situation.

Participants of our study watched two videos, one for each level of EI. The order in which the videos were presented to the participants was randomized. Each participant was assigned to either the involved or the distracted situation by chance.

We provided our participants with a questionnaire containing ten questions that can be rated using a five-point scale:

Q01: Is Christian helpful?

Q02: Is Louisa emotionally involved in the dialogue?

Q03: Does Christian plan his answers?

Q04: How responsive is Christian to Louisa's contributions?

Q05: Are Christian's answers spontaneous?

Q06: Is Christian emotionally involved in the dialogue?

Q07: How natural is the course of dialogue?

Q08: How much would you like to participate in such a dialogue?

Q09: Which dialogue is more natural?

Q10: In which conversation would you rather participate?

Q01-Q08 were asked for each the high EI-level and the low EI-level video. Those questions can be rated on a scale from 1-not at all to 5-very much. Q09 and Q10 compare the videos directly with each other and their scale is labelled from 1-high EI-level video to 5-low EI-level video.

\subsection{Results}

Our results show that both our research question can be answered in the affirmative. Multiple significant differences between the two EI-levels can be found, and the

\footnotetext{
${ }^{1}$ https://youtu.be/NRDIJ7omlEI

2 https://youtu.be/VqXgxFbh5-w

${ }^{3}$ https://youtu.be/3-ANJmmeZY

${ }^{4}$ https://youtu.be/bAXbt65vxjw
} 


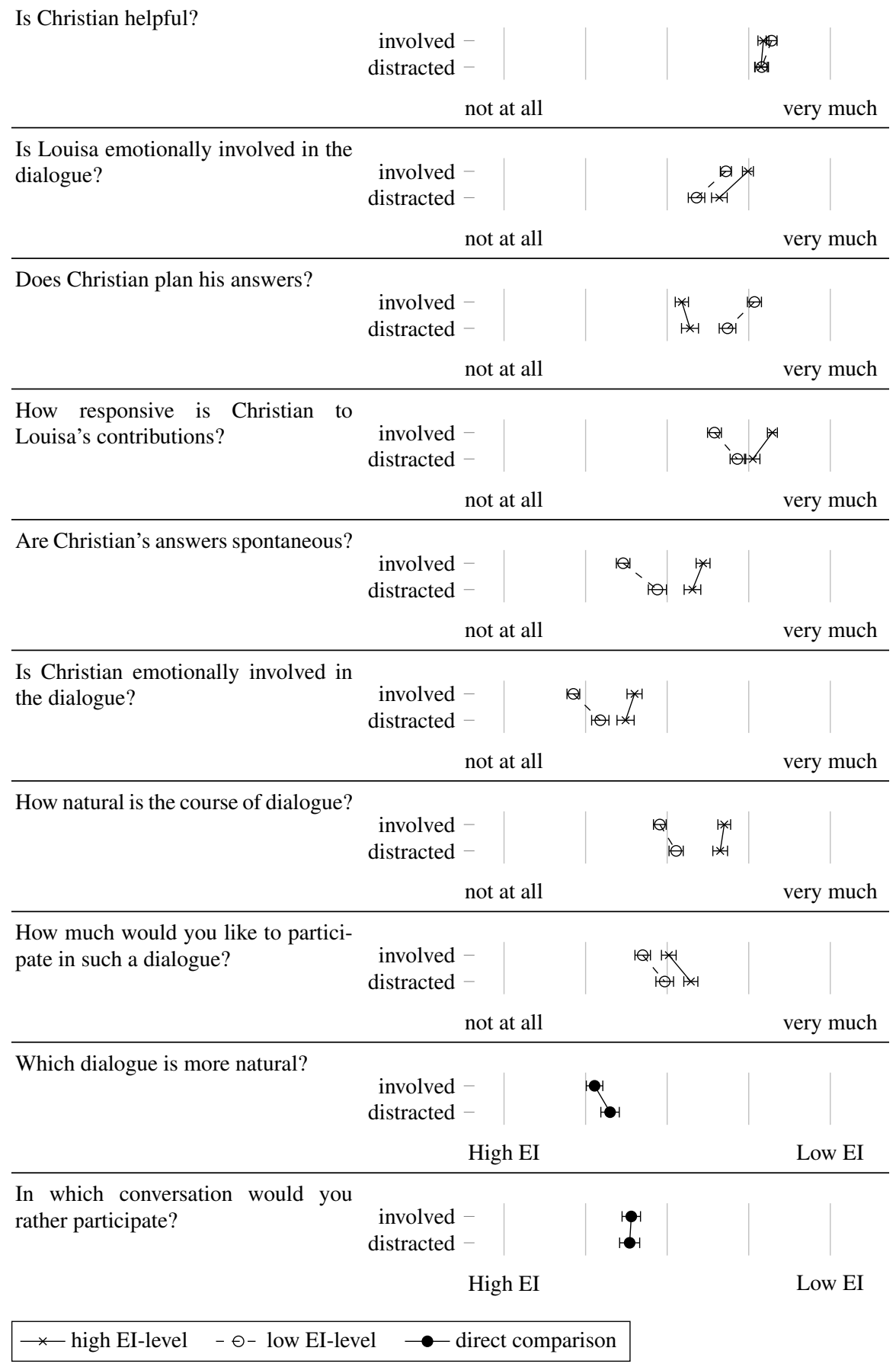

Fig. 2 Mean and standard error of the mean for the ratings of each question. 
assessment of EI-levels changes depending on the situation. Also, there are significant interaction effects between EI-level and situation. A graphical representation of the results can be found in Figure 2. In the following we present a more detailed description of the results.

Overall, 270 participants took part in our study. 154 participants filled in the questionnaire for the an involved user and 116 participants reported on the dialogue with the distracted user.

We test our hypotheses using T-test and ANOVA. Normal distribution of the data is assumed under the central limit theorem and homogeneity of variances is confirmed by Levene's test.

Slightly more women than men participated in the involved condition of the study (61 men, 87 women), and almost twice as many men as women in the distracted condition (74 men, 40 women). No significant effect of gender on the results of the study could be found. Most participants were between 20 and 29 years old (involved: $84.4 \%$, distracted: $72.4 \%$ ) and had only a limited amount of experience regarding dialogue systems (One time use or less: involved: $80.4 \%$, distracted: $60.4 \%$ ).

In all conditions, Christian is perceived as helpful. Changing the EI level does not negatively affect the ability of a dialogue system to help the user with their requests. All ratings for Q01 are significantly higher than $3(p<0.001)$.

Furthermore the actor portraying Louisa was perceived as involved in the involved condition, achieving a rating significantly higher than 3 for $\mathrm{Q} 02(p<0.001)$. In the distracted condition, Louisa did not succeed to appear sufficiently distracted, still receiving a rating significantly higher than $3(p<0.001)$. However, she at least appears less involved than in the involved condition, scoring a significantly lower rating $(p<0.001)$.

When Louisa is depicted as involved, participants rate Christian as more spontaneous, responsive, and emotionally involved if he uses a high EI-level and more planning ahead if he uses a low EI-level. Furthermore, the dialogue is perceived as more natural if a high EI-level is used, and participants would rather partake in such a dialogue. Significant differences can be detected for Q03-Q10 $(p<0.001$ in all cases). Those findings support the hypothesis that the EI-level has a strong impact on the user's perception of the dialogue partner as well as the overall dialogue. Furthermore, this perception changes as Louisa is perceived as less involved in the distracted condition. The EI-level no longer influences how responsive, spontaneous and emotionally involved Christian appears and how much participants would like to participate in the dialogue. Q04, Q05, Q06 and Q08 no longer show significant differences between the EI-level in the distracted condition of the study, and for Q04 and Q05, a significant interaction effect $(p<0.001)$ can be found between EIlevel and situation. This supports our second hypothesis that the effect of EI on the assessment of a dialogue changes in relation to the user's situation. 


\subsection{Virtual Avatar vs. Human Conversation Partner}

In addition to our main research questions, we compared a human and a virtual avatar as dialogue partners to check for potential differences of the impact of the EI-level in human-human and human-computer interaction.

Only Q06, 'Is Christian emotionally involved in the dialogue?', achieves a significant difference, with higher ratings for the human $(p<0.001)$. This might be due to a higher expressiveness of the human actor or to a reluctance of the participants to attribute involvement to a virtual agent.

\section{Implications for Adaptive Dialogue Management}

We could show in our study that the perception of a dialogue varies depending on the EI-level. This implies that the suitable EI-level should be considered in a dialogue system in order to provide a better user experience. A virtual avatar can portray different characteristics such as spontaneity or responsiveness by adjusting the EIlevel.

Our study also suggests that the effects of the EI-level depend at least to some degree on the situation of the user. Although the user was still perceived as being rather involved in the distracted condition, a significant difference to the involved portrayal was reported by the participants and resulted in a significant change of the assessment of the avatar's characteristics. The perceived difference between the EI-levels is less pronounced in the distracted condition of our user study.

We conclude that the level of EI can indeed be utilised by an adaptive DM, as the EI level influences the user experience and does so in different ways in different situations. Exemplary, we can conceive the following behaviour for a dialogue system from our results: if the user is involved in the dialogue, a high EI-level is employed to achieve the impression that the avatar is an empathic and spontaneous conversation partner. If the user becomes distracted, the EI-level does not influence the perceived characteristics of the avatar as much. Therefore, the dialogue system can employ varying degrees of EI to keep the dialogue more diversified and interesting. Furthermore, a more pronounced change in Louisa's situation (or other conditions such as emotion) might result in different user preferences regarding the level EI that can be taken into account by adaptive dialogue management, although this assumption will have to be tested in further studies.

\section{Conclusions and Future Work}

Most DMs pursue a hard approach to adaptation. In this paper, we investigated whether soft approaches, such as changing the level of EI, can be successfully applied in adaptive dialogue management. We presented a user study with which we 
were able to ascertain the impact EI on the assessment of a dialogue and show that this assessment changes in relation to the user's situation. We conclude from our results that EI offer valuable possibilities for adaptation and therefore should be incorporated in adaptive dialogue management.

In future work, the scope of applicability of EI, e.g. as means to adapt to emotion or culture, can be assessed. Furthermore, the automatic modification of dialogue actions with the goal to produce the desired level of EI should be investigated.

Acknowledgements This paper is part of a project that has received funding from the European Union's Horizon 2020 research and innovation programme under grant agreement No 645012.

\section{Appendix}

This appendix contains the complete dialogues spoken in the videos of the user studies for both the high EI and the low EI level, in order to enable a more extensive understanding of the conducted studies.

\section{Dialogue of the High EI Condition}

SYSTEM: Hello Louisa.

USER: Hello Christian.

SYSTEM: How can I help you?

USER: I'm afraid Mr. Smith doesn't drink enough during the day.

SYSTEM: How much does Mr. Smith usually drink?

USER: Only one cup of tea after breakfast.

SYSTEM: You're right, that is not enough. Do you know why he doesn't drink enough?

USER: He doesn't like the taste of water. He would rather have some soft drink, but I don't think that is good for him.

SYSTEM: Then you should try sweetened tea. It tastes good and is not as bad as a soft drink.

USER: Perhaps I don't even need to sweeten it. He does drink tea in the morning, after all.

SYSTEM: Exactly. A little company may help as well. While sitting and chatting you can easily drink some glasses without even noticing it.

USER: I guess that's true. Chatting is just more comfortable with a nice cup of tea.

SYSTEM: By the way, does Mr. Smith use a clear glass?

USER: Yes, he does. A beer glass with a handle, so he can better grab it. Why?

SYSTEM: That might be too unobtrusive. A clear glass filled with water does not really attract attention. 
USER: Do you think something more eye-catching would be better.

SYSTEM: It is worth a try. Perhaps something more colourful?

USER: I could use the cup his granddaughter gave him. It's very colourful.

SYSTEM: That is a very good idea. He will be happy whenever he sees that cup. USER: I think so, too. He loves her very much. Also, that cup has a handle. That

is very important for him, he feels more secure when grabbing something with a handle.

SYSTEM: Many elderly feel that way.

USER: But what if Mr. Smith still doesn't drink enough? After all, I need to increase that quite a lot.

SYSTEM: The body doesn't only get fluid by drinking. Food contains fluid too. USER: I don't think that will be enough.

SYSTEM: There some dishes that contain a lot of fluid, such as soup.

USER: Or goulash? He really likes that.

SYSTEM: Yes, goulash is fine. And fruits.

USER: Alright. Thank you, Kristina. You really helped me.

SYSTEM: No problem at all.

USER: I'll get back to work.

SYSTEM: I will be here if you need me.

\section{Dialogue of the Low EI Condition}

SYSTEM: Hello Louisa.

USER: Hello Christian.

SYSTEM: How can I help you?

USER: I'm afraid Mr. Smith doesn't drink enough during the day.

SYSTEM: How much does Mr. Smith usually drink?

USER: Only one cup of tea after breakfast.

SYSTEM: You're right, that is not enough. Do you know why he doesn't drink enough?

USER: He doesn't like the taste of water. He would rather have some soft drink, but I don't think that is good for him.

SYSTEM: Then you should try sweetened tea. It tastes good and is not as bad as a soft drink.

USER: Perhaps I don't even need to sweeten it. He does drink tea in the morning, after all.

SYSTEM: Yes, you can try that.

USER: But what if Mr. Smith still doesn't drink enough? After all, I need to increase that quite a lot.

SYSTEM: I have got three more ideas. Do you want to hear all of them?

USER: Yes, please.

SYSTEM: First, you should keep him company. While chatting people usually drink a lot without even realising it. 
USER: I guess that's true. Chatting is just more comfortable with a nice cup of tea.

SYSTEM: Second, you can increase his fluid intake by preparing dishes that contain much fluid, such as soup, goulash and fruits.

USER: He really likes goulash.

SYSTEM: Does Mr. Smith use a clear glass?

USER: Yes, he does. A beer glass with a handle, so he can better grab it. Why?

SYSTEM: In that case you should give him a more colourful glass. A clear glass

filled with water does not really attract attention.

USER: I could use the cup his granddaughter gave him. It's very colourful.

SYSTEM: Then it is a good choice.

USER: Alright. Thank you, Kristina. You really helped me.

SYSTEM: No problem at all.

USER: I'll get back to work.

SYSTEM: I will be here if you need me.

\section{References}

1. André, E., Rehm, M., Minker, W., Bühler, D.: Endowing spoken language dialogue systems with emotional intelligence. In: Affective Dialogue Systems, pp. 178-187. Springer (2004)

2. Bertrand, G., Nothdurft, F., Minker, W., Traue, H., Walter, S.: Adapting dialogue to user emotion-a wizard-of-oz study for adaptation strategies. Proc. of IWSDS pp. 285-294 (2011)

3. Chu-Carroll, J.: Mimic: An adaptive mixed initiative spoken dialogue system for information queries. In: Proceedings of the sixth conference on Applied natural language processing, pp. 97-104. Association for Computational Linguistics (2000)

4. Damian, I., Endrass, B., Huber, P., Bee, N., André, E.: Individualizing Agent Interactions. In: Proc. of 4th Int. Conf. on Motion in Games (MIG 2011) (2011)

5. Gnjatović, M., Rösner, D.: Adaptive dialogue management in the NIMITEK prototype system. In: Perception in Multimodal Dialogue Systems, pp. 14-25. Springer (2008)

6. Jaksic, N., Branco, P., Stephenson, P., Encarnação, L.M.: The effectiveness of social agents in reducing user frustration. In: CHI'06 extended abstracts on Human factors in computing systems, pp. 917-922. ACM (2006)

7. Kousidis, S., Kennington, C., Baumann, T., Buschmeier, H., Kopp, S., Schlangen, D.: A multimodal in-car dialogue system that tracks the driver's attention. In: Proceedings of the 16th International Conference on Multimodal Interaction, pp. 26-33. ACM (2014)

8. Saerbeck, M., Schut, T., Bartneck, C., Janse, M.D.: Expressive robots in education: varying the degree of social supportive behavior of a robotic tutor. In: Proceedings of the SIGCHI Conference on Human Factors in Computing Systems, pp. 1613-1622. ACM (2010)

9. Turunen, M., Hakulinen, J.: Agent-based adaptive interaction and dialogue management architecture for speech applications. In: Text, Speech and Dialogue, pp. 357-364. Springer (2001)

10. Ultes, S., Minker, W.: Managing adaptive spoken dialogue for intelligent environments. Journal of Ambient Intelligence and Smart Environments 6(5), 523-539 (2014) 